\title{
TYPES AND CONSTRUCTION OF POWER TRANSFORMERS
}

\section{Introduction}

Transformers may be classified according to dielectric insulation material as follows:

- Oil-filled transformers

- Dry type transformers

\section{Oil-filled Transformers}

Two types of oil-filled transformers are commonly used:

- With expansion tank (conservator)

- Sealed

In this type of transformers windings and core are immersed in oil, in a tank with radiators; oil plays both functions of insulating material and cooling fluid.

Common applications of oil-filled transformers with conservator are:

- As step-up transformers in power plants.

- As step-down transformers in EHV/EHV, EHV/HV and HV/MV utilities substations (primary voltages above $52 \mathrm{kV})$.

- As step-down transformers in $\mathrm{HV} / \mathrm{MV}$ or $\mathrm{MV} / \mathrm{MV}$ industrial plants substations, with rated power above 2.5 MVA.

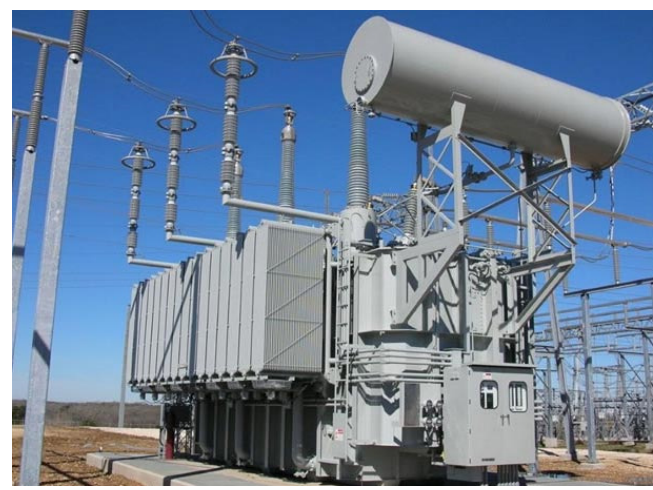

Oil-filled sealed transformers (without conservator) are mainly used in distribution networks (MV/LV) and in installations up to $52 \mathrm{kV}$, with a rated power up to $2.5 \mathrm{MVA}$, although some manufactures built this type of transformers up to 30 MVA.

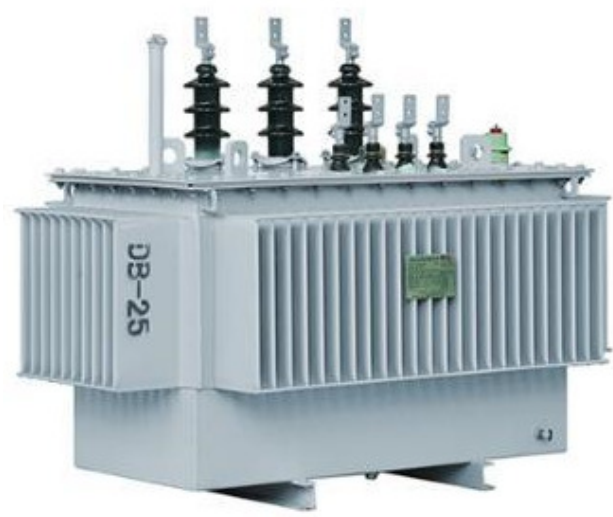

The degree of protection (IP) provided by the tank allows that both types of transformers can be installed outdoors.

\section{Dry Type Transformers}

Dry type transformers present the most suitable solution in situations where the distribution of energy requires absolute safety and environmental friendliness. These transformers require less maintenance than oil-filled transformers, more safe to environment and have low fire hazard.

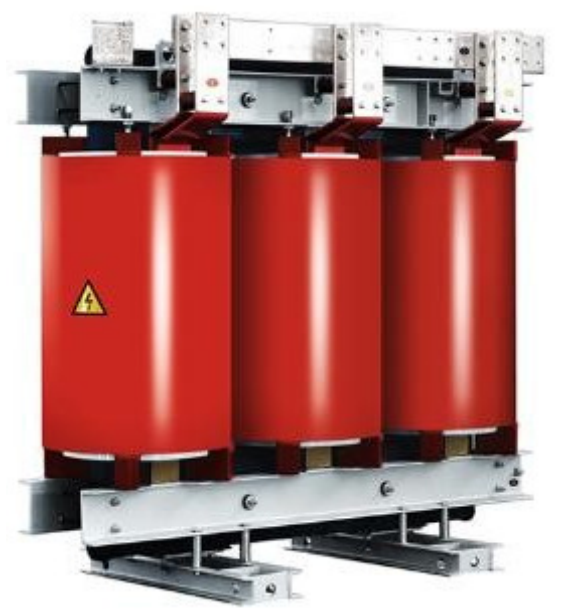

Windings and core are not installed in a tank and insulation of windings is usually made of cast resin.

They possess less space, about $2 / 3$ of that of corresponding oil filled transformers, and their simple construction allows on-site replacement of windings. 
Dry type transformers are only suitable for indoors installation because the degree of protection provided by enclosure is IPOO and are usually employed on distribution networks and in installations with voltages up to $52 \mathrm{kV}$ and rated power up to $2.5 \mathrm{MVA}$, although some manufactures built this type of transformers up to 50 MVA.

Typical use of dry type transformers is:

- Multi-storey buildings

- Hotels

- Malls

- Hospitals and clinics

- Airports

- Mines

- Other places where fire safety is a great concern

\section{Gas Insulated Transformers (GIT)}

Rising demand for electric power in large cities, lack of space to construct new substations or upgrade existing substations and adverse environment conditions has encouraged largescale substations to be tucked away underground in overpopulated urban areas, leading to strong demand for incombustible and non-explosive, large-capacity gas insulated transformers from the view point of accident prevention and compactness of equipment.

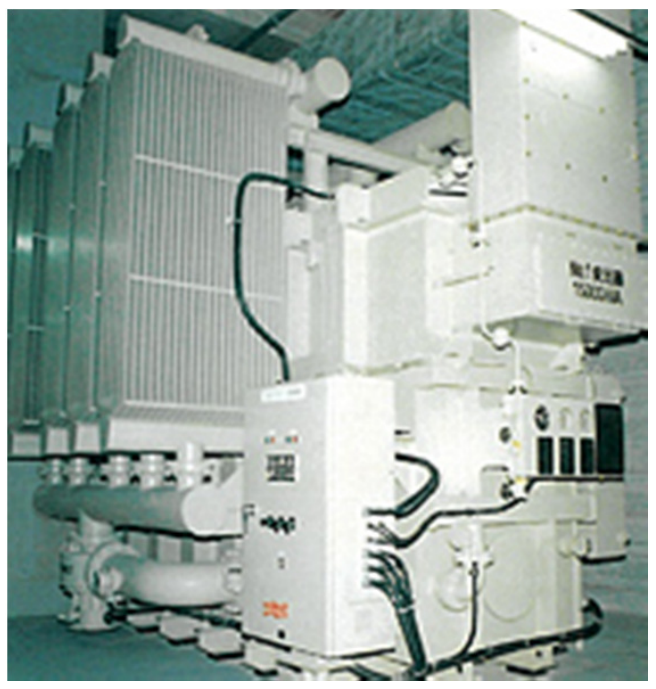

In line with this requirement, several types of large-capacity gas insulated transformers have been developed, being SF6 the most common gas used.
Because the gas forced cooling type was considered to be available up to approximately 60 MVA, all of these gas insulated transformers are liquid cooled.

The main advantages of GIT are:

- Non flammable (gas insulated transformers, using incombustible SF6 gas as an insulation and cooling medium).

- Explosion-proof tank (pressure tank withstands pressure rise in case of internal fault).

- Compactness (since conservator or pressure relief equipment is not necessary, height of transformer room can be reduced approximately 2-2.5 meters).

- Easy installation (oil or liquid purifying processes are not necessary with gas insulated transformers).

- Easy inspection and maintenance work (only SF6 gas pressure need be basically monitored during periodic inspection).

Since gas insulated transformers do not need a conservator, the height of transformer room can be reduced. In addition, its non-flammability and non tank-explosion characteristics can remove firefighting equipment from transformer room.

As a result, gas insulated transformers, gas insulated shunt reactor, GIS and control panels can be installed in the same room, and such installation realizes the fully SF6 gas insulated substation.

\section{Two and Three Windings Transformers}

Usually transformers have two windings, the primary and the secondary.

However, in HV and EHV substations, with voltages above 52 $\mathrm{kV}$, power transformers may have a third winding, with a rated voltage of $7 \mathrm{kV}$ or $11.5 \mathrm{kV}$.

This winding is used for harmonic compensation, to reduce the unbalancing in the primary due to unbalancing in three phase load and to redistribute the flow of fault current. It is common practice to use this third winding to LV auxiliary services power transformer. 


\section{Single Phase Transformers and Autotransformers} For voltages above $123 \mathrm{kV}$ and for high values of rated power (usually above $100 \mathrm{MVA}$ ), for handling and economic reasons it is usual to use single phase transformers.

In such a situation the three single phase transformers must work as a whole, since they are relatively interdependent.

The windings (both primary and secondary) of the three single phase transformers must be connected together in "star" or in "delta".

A special case of single phase transformers are autotransformers that have one single core and winding.

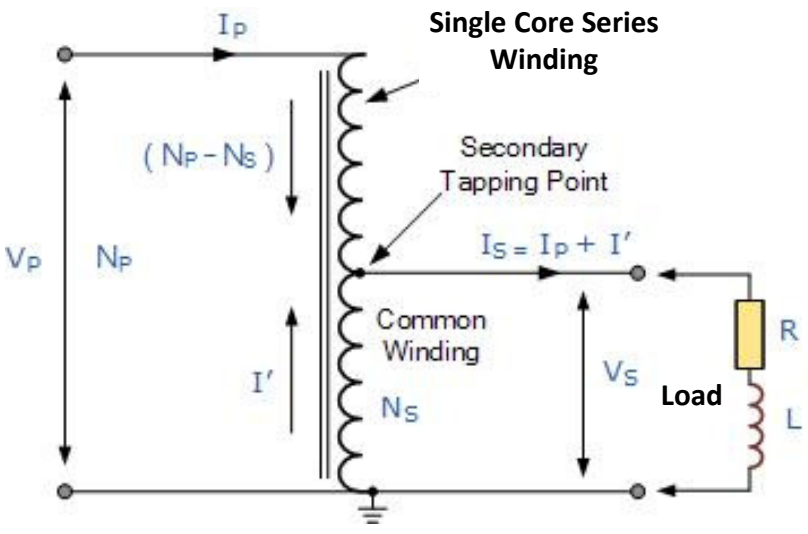

The main advantages of an autotransformer compared to a common single phase transformer are:

- More economic and easier to handle (only one winding and for the same rated power the dimensions and the weight are lower).

- More efficient (losses by Joule effect are lower, because there is only one winding)

- Lower voltage drop, being able to keep the voltage more stable.

In contrast the major disadvantages are:

- Primary and secondary windings are not isolated from each other.

- As the internal voltage drop is lower, in case of a short circuit the fault current is higher, causing higher electrodynamics stress in the windings, which by be a cause of ageing.

\section{Dissociated Phases Transformers}

Another construction solution for voltages above $123 \mathrm{kV}$ and for high values of rated power is the dissociated phases transformer, formed by three single-phase transformers within a common enclosure, where are made the connections of the three transformers and where the bushings are assembled.

The conservator, the cooling system and the on-load tap changer may be also assembled at that enclosure or assembled separately.

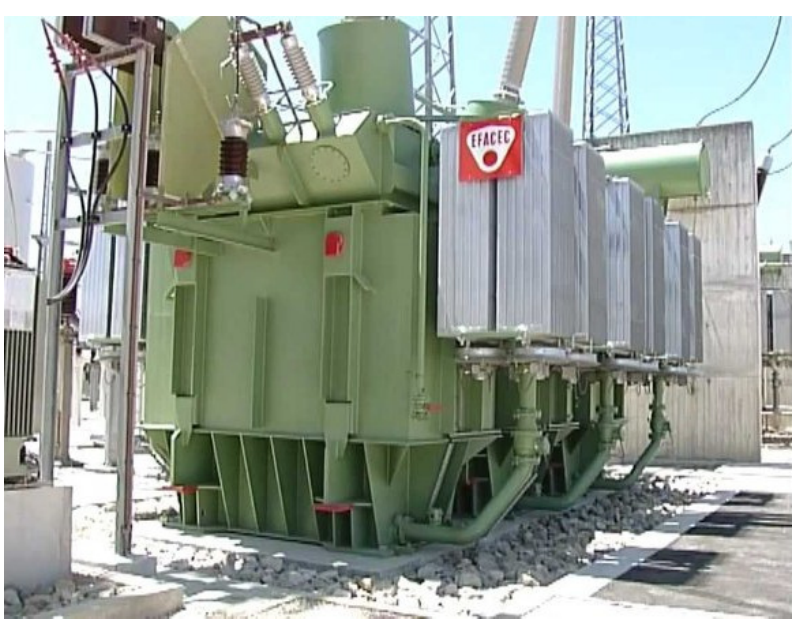

\section{Zig-Zag \& Grounding Transformers}

Neutral grounding of transformers and transmission and distribution networks may be:

- Solid grounded

- Grounded through an impedance or a resistance

- Ungrounded

When it is required to connect the neutral of the transformers to the ground, (solid or through an impedance or a resistance) and when at the side it is designated to ground the neutral the windings are "delta" connected (usually $\mathrm{MV} / \mathrm{LV}$ power transformers), in order to achieve neutral grounding is necessary to form an artificial neutral point. This is achieved using a zigzag grounding transformer. 